\title{
Une catéchiste québécoise : Soeur Saint-Ladislas, a.s.v.
}

\section{Thérèse Bernier}

Volume 52, 1985

URI : https://id.erudit.org/iderudit/1007004ar

DOI : https://doi.org/10.7202/1007004ar

Aller au sommaire du numéro

Éditeur(s)

Les Éditions Historia Ecclesiæ Catholicæ Canadensis Inc.

ISSN

0318-6172 (imprimé)

1927-7067 (numérique)

Découvrir la revue

Citer cet article

Bernier, T. (1985). Une catéchiste québécoise : Soeur Saint-Ladislas, a.s.v.

Sessions d'étude - Société canadienne d'histoire de l'Église catholique, 52, 77-87.

https://doi.org/10.7202/1007004ar

\section{Résumé de l'article}

Le Congrès se tenant à la Maison-Mère des Soeurs de l'Assomption de la Sainte Vierge, la Société a voulu présenter une communication sur cet Institut. On trouvera ici un témoignage sur Soeur Saint-Ladislas, religieuse de la Congrégation, pionnière du renouveau catéchétique au Québec dans les années 1935-1950.

Après un doctorat en pédagogie et des études en théologie, en littérature et en histoire, Soeur Saint-Ladislas mit de l'avant une méthode inductive intégrale d'explication du catéchisme, dans une série de six volumes intitulés Aux Petits $d u$ Royaume. Elle eut une influence déterminante dans la réforme des programmes de catéchèse de l'après-guerre.
Tous droits réservés @ Les Éditions Historia Ecclesiæ Catholicæ Canadensis Inc., 1985
Ce document est protégé par la loi sur le droit d'auteur. L'utilisation des services d'Érudit (y compris la reproduction) est assujettie à sa politique d'utilisation que vous pouvez consulter en ligne.

https://apropos.erudit.org/fr/usagers/politique-dutilisation/ 


\section{Une catéchiste québécoise: Soeur Saint-Ladislas, a.s.v.*}

Thérèse BERNIER, s.a.s.v.,

Nicolet, Qué.

\section{RÉSUMÉ}

Le Congrès se tenant à la Maison-Mère des Soeurs de l'Assomption de la Sainte Vierge, la Société a voulu présenter une communication sur cet Institut. On trouvera ici un témoignage sur Soeur Saint-Ladislas, religieuse de la Congrégation, pionnière du renouveau catéchétique au Québec dans les années 1935-1950.

Après un doctorat en pédagogie et des études en théologie, en littérature et en histoire, Soeur Saint-Ladislas mit de l'avant une méthode inductive intégrale d'explication du catéchisme, dans une série de six volumes intitulés Aux Petits du Royaume. Elle eut une influence déterminante dans la réforme des programmes de catéchèse de l'après-guerre.

«Un grand fait apparaît en plein jour dans l'histoire du Canada, c'est l'Église de Rome. Plus encore que le pouvoir royal, elle a modelé le caractère et le destin de la colonie» ${ }^{1}$.

\footnotetext{
* Nous avons profité du travail de Soeur Anita Sylvestre, a.s.v. Une pionnière du renouveau catéchistique (texte polycopié). Il s'agit là d'une excellente source de références sur Soeur Saint-Ladislas.

${ }^{1}$ F. Parkman, The Old regime in Canada, Boston, Little et Brown, 1895, p. 450.
} 
Lorsque l'historien Parkman s'exprimait ainsi, l'Église catholique catéchisait depuis environ deux siècles au Canada. La catéchèse de saint Jean de Brébeuf et des autres missionnaires avait formé des mystiques tels Catherine Tékakouita, et des disciples fervents parmi les colons de la Nouvelle-France. Ni les missionnaires ni l'Église ne se contentent du travail accompli. On veut toujours perfectionner la catéchèse. Le catéchisme de Brébeuf influence celui de $\mathbf{M}^{\mathrm{gr}}$ de Saint-Vallier en raison du précieux avantage d'offrir à la fois, la théorie et la pratique.

Pas un instant les Évêques de la Nouvelle-France ne perdent de vue le développement de la doctrine chrétienne. Un mandement de $\mathbf{M}^{\mathrm{gr}}$ Briand, daté de 1777, rend officiel au Canada le catéchisme français de $\mathbf{M}^{\mathrm{gr}}$ Languet, appelé aussi Catéchisme de Sens ${ }^{2}$. Quelque peu adapté au Canada, le Catéchisme prend le nom de Catéchisme de Sens-Québec. Il a cours au Canada jusqu'en 1815, en dépit des critiques des prêtres et des laïcs. On n'a pas sur place les moyens d'une réforme immédiate. Cependant, l'Église prépare une réforme de ce dernier manuel qui conduit à l'approbation en 1815, par $\mathrm{M}^{\mathrm{gr}}$ Plessis, d'un traité amélioré, intitulé «Le Petit Catéchisme».

Les évêques profitent du Premier Concile provincial de Québec pour mettre au point une nouvelle édition catéchistique, diffusée en 1853, sous le titre de Petit Catéchisme de Québec. Un mandement collectif, daté du 8 septembre 1853 et signé par les sept évêques de la province ecclésiastique, est envoyé au clergé régulier et séculier ainsi qu'aux fidèles de la province. Ce mandement manifeste l'importance attachée par l'Église à la catéchèse du peuple canadien.

Le Petit Catéchisme de Québec, officiel le 8 septembre 1853, date de la fondation des Soeurs de l'Assomption, est donc le premier catéchisme utilisé par nos fondatrices. Faudrait-il faire remonter à cette époque le charisme d'enseignement religieux attribué à notre Congrégation? Pour l'explication du nouveau catéchisme, nos fondatrices ont profité des cours de religion de deux pédagogues: M. l'abbé Harper qui s'était fait remarquer dans l'Ouest par son évêque, $\mathbf{M}^{\mathrm{gr}}$ Provencher, et $\mathbf{M}$. l'abbé Marquis, professeur au Séminaire de Québec avant d'être vicaire à St-Grégoire. Tous deux se sont occupés de la formation religieuse des Soeurs et de la catéchèse des élèves de St-Grégoire, dès 1853.

${ }^{2}$ Fernand Porter, L'Institution catéchistique au Canada français, 1633-1833, Montréal, les Éd. franciscaines, 1944, p. 111. 
Au Petit Catéchisme de Québec, succède, en 1888, le Catéchisme des provinces ecclésiastiques de Québec, Montréal et Ottawa. Dans les Archives de la Maison mère, on conserve le minuscule volume portant le nom de Soeur Saint-Ladislas. C'est afin de rendre plus vivante la lettre de ce catéchisme que la religieuse est entrée, vers 1935, au coeur d'un mouvement de rénovation catéchistique. Qui est Soeur Saint-Ladislas? En quoi consiste son oeuvre? Quelle en a été l'influence? Ce sont là les questions auxquelles on tentera de répondre brièvement.

\section{Qui est Soeur Saint-Ladislas?}

Marguerite Gauthier est née en 1897 à Saint-Bruno-de-Guigues, comté de Témiscamingue. À l'âge de six ans, elle fréquente l'école du rang. L'incompréhension de certaines formules de catéchisme suscite chez elle des malaises profonds. Madame Gauthier provoque une confidence et calme vite les inquiétudes de sa fillette ${ }^{3}$. Cet événement exerce une influence capitale sur la vocation d'éducatrice et d'auteur de Marguerite.

Après avoir poursuivi ses études au couvent des Soeurs de l'Assomption de Saint-Bruno-de-Guigues et enseigné quelques années à l'école du rang, elle entre, en 1918, au Noviciat de la même Congrégation, où, par la suite, elle sait faire fructifier les dons d'une nature généreuse à son endroit. Quel est donc le visage de cette catéchiste voilée sous le pseudonyme «une religieuse de l'Assomption»? Nous croyons l'avoir décelé dans ses ouvrages, dans son enseignement, à travers les nombreux témoignages venus d'évêques, de religieux, de laïcs, d'éducateurs et d'anciennes élèves. Le premier trait de cette personnalité remarquable réside dans une science théologique approfondie appuyée sur une culture générale étendue. Soeur Saint-Ladislas sait qu'en matières religieuses, on ne peut se permettre d'erreurs. Elle sait aussi qu'une science se simplifie dans la mesure où elle est comprise; c'est pourquoi elle se livre à l'étude et y convie tous les éducateurs:

Il n'est pas dans le monde où nous vivons d'apostolat efficace sans culture vraie. Au service du Royaume, nous ne devons pas mettre seulement tout notre amour; il nous faut forger les armes subtiles de l'esprit, devenir des instruments de choix, riches de dons humains ${ }^{4}$.

\footnotetext{
${ }^{3}$ Luce Dumoulin, Étude bio-bibliographique, Soeur Saint-Ladislas et son oeuvre d'enseignement religieux. Archives de la Maison mère de Nicolet (désormais AMMN).

${ }^{4}$ Soeur Saint-Ladislas, Notes manuscrites d'un cours donné à l'Université Laval, Québec, juillet 1944. AMMN.
} 
C'est parce qu'elle est femme d'étude d'un savoir éprouvé qu'elle distingue, analyse, éclaire et synthétise les connaissances. Elle fait jaillir la vie des formules inertes; elle guide les jeunes intelligences dans le monde des réalités religieuses; elle utilise la philosophie, la théologie. la pédagogie au service non pas d'une matière mais de la religion qui transcende les autres matières.

Que dire maintenant de sa psychologie de l'âme enfantine? Elle s'adapte au développement intellectuel de ses jeunes auditeurs: elle découvre leurs intérêts, elle présente ce qui captive leur coeur et leur imagination. Par ses jugements et ses attitudes, elle s'applique à établir en eux une hiérarchie des valeurs. Elle relativise l'importance des points, des rangs ${ }^{5}$. de l'argent ${ }^{6}$, des toilettes ${ }^{7}$. En plus d'être de fines mises au point, ses leçons concrétisent la doctrine chrétienne. Soeur Saint-Ladislas pédagogue, la plupart des critiques le reconnaissent. $\mathrm{M}^{\mathrm{gr}}$ Desranleau, évêque de Sherbrooke, s'exprime ainsi: «La partie pédagogique est très bien, les enfants peuvent suivre, c'est à leur portée» ${ }^{8}$. $\mathbf{M}^{\mathrm{gr}}$ Forget découvre la même qualité: «Je m'empresse de féliciter l'auteur... d'un grand talent de vulgarisation de l'enseignement religieux»" ${ }^{9} \mathrm{M}^{\mathrm{gr}}$ Tessier, à son tour encourage:

Une Somme catéchistique à la portée de tous, vivante, lumineuse, chargée de doctrine. Même les adultes y compléteront, y rajeuniront leurs connaissances religieuses trop souvent noyées dans une terminologie vidée de son sens réel. Qu'ils relisent, avec une âme rajeunie, ces textes pleins d'images, de comparaisons, de traits tirés de la vie de tous les jours. Ils y découvriront, avec infiniment de joie, l'extraordinaire richesse de ces pages qui empruntent à l'Évangile des procédés directs que les parents, les éducateurs et même les prédicateurs avaient perdu $^{10}$.

Ces témoignages d'évêques et d'autres que nous ne pouvons citer, en raison de leur nombre, nous disent le talent de pédagogue et de psychologue de Soeur Saint-Ladislas.

À une physionomie déjà belle des dons multiples énumérés, ajoutons une âme poète, vibrant à la beauté des valeurs spirituelles et soucieuse de

\footnotetext{
5 Soeur Saint-Ladislas, Aux Petits du Royaume, Trois-Rivières, Éd. du Bien Public, 1945 , p. 219.

6 Ibid., p. 103.

7 Ibid., p. 31.

8 P. Desranleau à la Maîtresse des Études, 8 mars 1941, AMMN.

9 A. Forget à la Maîtresse des Études, AMMN.

10 Albert Tessier, «Un ouvrage qui nous vient comme un cadeau providentiel», Bulletin des Écoles ménagères, n 42, juin 1942, p. 2.
} 
les faire aimer aux autres. Sa sensibilité religieuse se plaît à découvrir aux enfants les secrets de l'amour du Père, dans la splendeur du soleil, dans la simplicité des fleurs champêtres, dans le chant des oiseaux, dans l'agilité de l'écureuil ou encore devant les champs d'avoine et les plantureux potagers. Lorsqu'elle utilise la magie des beautés naturelles pour évoquer le surnaturel, c'est avec un profond sentiment de foi religieuse, contagieux pour ses auditeurs. On entend alors le silence appeler au dépassement; elle devient un éclaireur sur la route du Royaume. Les titres de ses livres évoquent son esprit surnaturel.

Soeur Saint-Ladislas est aussi une femme de son temps; elle épouse les souffrances d'un peuple en état de crise économique puis en état de guerre. On dirait que parfois une claire vision de l'avenir la projette vers les chemins nouveaux de l'adaptation: adaptation au sujet de l'enseignement, à son objet et à son temps. Lorsque le 31 décembre 1929, le Pape Pie XI s'adresse au monde par l'encyclique sur l'éducation, Soeur SaintLadislas prend le texte et l'annote (on retrouve cet exemplaire aux Archives de la Maison mère). Elle en fait le sujet d'un cours au Noviciat. Les notes manuscrites de ces cours disent l'attachement de Soeur SaintLadislas aux directives ecclésiales. En janvier 1934, le cardinal Villeneuve lançait un cri d'alarme devant le Cercle universitaire de l'Université de Montréal: "Ce n'est pas d'ignorer une théologie élémentaire que je vous reproche, c'est d'ignorer le petit catéchisme»" ${ }^{1}$. Ce reproche aux universitaires, Soeur Saint-Ladislas le rappelle aux éducateurs pour éveiller chez eux un souci d'approfondissement des connaissances, et une objective remise en question de la valeur de leur méthode d'enseignement ${ }^{12}$. Ce que nous venons de voir de sa personnalité, Soeur Saint-Ladislas l'exprime dans son oeuvre principale, Aux Petits du Royaume, méthode d'introduction vivante et synthétique de pédagogie de la catéchèse.

\section{L'Oeuvre d'une catéchète: une méthode de pédagogie catéchistique}

Certes, nous n'attribuerons pas à notre excellente catéchète l'invention ni de l'induction ni de la déduction, mais d'avoir tiré du neuf de l'ancienne méthode inductive, cela ne s'était pas encore vu dans la catéchèse canadienne-française. Afin de mieux préparer l'esprit des enfants à recevoir la doctrine abstraite du christianisme, Soeur Saint-Ladislas utilise le

11 J.M.R. cardinal Villeneuve, Quelques pierres de doctrines, Montréal, Beauchemin, 1938, p. 100.

12 Soeur Saint-Ladislas, Catéchisme et vie chrétienne, Trois-Rivières, Éd. du Bien Public, 1945, p. 49. 
concret. Elle puise aux multiples sources de la nature qui entoure les enfants; elle invente des récits qui captivent l'imagination et se sert aussi de l'Écriture sainte; ses leçons abondent en comparaisons et en exemples pris dans la vie des jeunes. Comment arrive-t-elle à cette méthode? Éducatrice-née et femme d'étude, elle observe, elle cherche dans les carences de l'enseignement religieux sans cesse décrié et dans l'absence de pédagogie catéchistique les causes d'une ignorance manifeste dans toutes les classes de la société.

Elle n'est pas femme à demeurer inactive face aux problèmes de son peuple. Mais le seul moyen à sa portée, pour remédier à tout cela, c'est de tirer profit de sa propre expérience; elle tente de canaliser, au bénéfice de la science catéchistique, les ressources de la philosophie et de la pédagogie moderne. Chaque leçon des six volumes Aux Petits du Royaume constitue un mode d'application de la méthode inductive.

Pourquoi a-t-elle écrit cet ouvrage? Par sympathie pour les institutrices du cours primaire. Ayant elle-même oeuvré dans les classes à divisions multiples, elle en connaît les inconvénients. De plus, chargée de cours au Noviciat, en septembre 1934, elle peut expérimenter toutes ses leçons et préparer ainsi de futures catéchètes. Elle sera contestée, même dans sa propre maison: pourquoi retourner au petit catéchisme, après sept ans d'études supérieures! La religieuse s'engage quand même à remédier aux misères de notre enseignement. À la dispersion, elle oppose la synthèse, la religion étant un tout; à la passivité, elle substitue la vie. Pour elle, être chrétien ne signifie surtout pas réciter des formules de mémoire, mais parvenir à une relation filiale avec le Père qui nous découvre ses secrets dans un amour paternel si grand! Connaître sa religion, c'est expérimenter des relations fraternelles avec Jésus, des relations avec la famille de Jésus, tous les membres de son corps...

Pourquoi écrit-elle ses volumes aux titres de lumière: Les secrets $d u$ bon Dieu - Les sources de la vie - La route dans la lumière. Pourquoi? Pour l'avènement du règne du Christ, ici et dans le monde, pour aider tous les catéchètes et tous les enfants. Sa vie n'est pas dominée par un désir de salut personnel; ah! sans doute, parle-t-elle de la béatitude promise, elle l'espère aussi, mais non pour elle seule. Au contraire, sa vie est un amour du Père; son oeuvre, un dévouement à Jésus-Christ, à qui elle s'est vouée. Elle ne vit pas pour les ovations, les félicitations, les louanges. Elle voudrait ne pas sortir de sa Congrégation. Elle souffre de devoir prononcer des séries de conférences. Ses Soeurs novices sont les premières à bénéficier de son travail. Les leçons du noviciat deviennent des modèles qu'on va expérimenter à la "petite école». Le regard observateur de la catéchiste- 
pédagogue a vite décelé le feu sacré allumé chez ses Soeurs et découvert des dispositions à la catéchèse dans sa propre Congrégation. Cela la réjouit profondément.

À l'expérience chez les élèves des $3^{\mathrm{e}}$ à $6^{\mathrm{e}}$ années du Pensionnat de Nicolet, les leçons de la catéchète passent par l'épreuve. Soeur SaintLadislas se corrige sans indulgence. Un exemplaire du premier volume Aux petits du-Rovaume conservé aux Archives, porte, en marge, la trace du labeur méthodique de l'auteur: «La leçon ne marche pas... fatigue des élèves... maladresse de ma part» ${ }^{13}$. Chacune de ses leçons dans les six volumes est expérimentée de la sorte, par elle-même, avant d'être livrée à l'éditeur:

Notre ambition unique, écrit-elle, étant de servir, ce n'est qu'après avoir mis la main à la pâte et combien de fois! et combien longtemps que nous nous sommes décidée à écrire les pages qui vont suivre et qui ne sont qu'une relation d'expériences vécues par nous-mêmes ${ }^{14}$.

Pour stimuler l'activité des élèves, Soeur Saint-Ladislas a aussi rédigé deux cahiers d'exercices pratiques sur l'ensemble de la doctrine. Le même souci d'expérimentation préside à l'élaboration des cahiers. Elle ne considère jamais son oeuvre terminée: la voilà en train d'exécuter une recherche auprès des élèves des $1^{\text {re }}$ et $2^{\mathrm{e}}$ années élémentaires. Le travail, poursuivi malgré la maladie, donne huit livrets Les tous-petits dans le Royaume. On imprime 1500 copies de chaque livret, on les distribue gratuitement à Trois-Rivières, Gentilly, Drummondville, Warwick, St-Léonard et La Baie-du-Febvre. Elle-même se rend sur les lieux, en septembre 1945, pour lancer l'expérience. À la fin de l'année scolaire, l'essai s'étant révélé concluant, les autorités décident de la publication des huit livrets de lectures catéchistiques. Après toutes ces expériences, notre catéchète écrit un ouvrage de réflexion pédagogique: Catéchisme et vie chrétienne.

Soeur Saint-Ladislas devient la femme-ressource: on l'invite ici pour un programme, là pour un article de revue, ailleurs on lui demande une conférence; elle appelle cette époque sa vie publique. Tenue de demander à son évêque le permis d'imprimer, elle lui parle de son dégoût des applaudissements. On dirait qu'elle redoute leurs séductions. Voici l'admirable réponse de $\mathrm{M}^{\mathrm{gr}}$ Albini Lafortune:

Que la différence entre le «paraître» et «l'être» ne vous accable pas trop. N'oubliez pas que l'idéal c'est le Christ; la réalité, c'est notre pauvre nous. Tout de même «l'Idéal» s'est complu avec la réalité. Pensez sou-

13 Aux Petits du Royaume, t. 1, 1939, AMMN, p. 9.

${ }^{14}$ Soeur Saint-Ladislas, Catéchisme et vie chrétienne. 
vent au bonheur que goûtait Jésus à s'arrêter à Béthanie. Ces haltes, si divinement humaines, nous ont valu les plus belles pages de l'Évangile. Le coeur de Jésus n'a pas changé. Il se complaît toujours avec ceux qui l'aiment et tâchent de le faire aimer. Entendez, vous aussi, le «bene scripsisti de me», non pour en tirer vanité, non plus pour être accablée. mais pour rendre gloire au Christ qui a fait par vous de grandes choses. Et comme saint Thomas, ne désirez pour récompense que la possession plus parfaite de Jésus-Christ. Puis après cela. chantez comme Marie: Magnificat anima mea Dominum ${ }^{15}$.

Outre ce précieux encouragement de son Évêque, Soeur SaintLadislas recevait aussi l'appui moral de $\mathrm{M}^{\mathrm{gr}}$ Saint-Germain, un vieil ami de la Congrégation, qui lui faisait un devoir d'écrire, de parler...

Une religieuse artiste, Soeur Monique Crête, a illustré les volumes et les cahiers; elle a soutenu elle aussi notre Soeur Catéchète qui pouvait toujours tout attendre d'elle à n'importe quel moment. Une autre religieuse, Soeur Marie-Immaculée, lui fut aussi d'un grand secours en relisant ses textes; dans l'état de santé où était Soeur Saint-Ladislas, c'était un soulagement et un appui. C'est ainsi qu'étant déjà initiée au travail, Soeur MarieImmaculée put le terminer après le départ de Soeur Saint-Ladislas.

Le Comité catholique du Département de l'Instruction publique lui confie, au secteur de la religion, la rédaction des Directives tant particulières que générales à chaque année du Cours élémentaire. La revue pédagogique, l'Enseignement primaire, lui demande une série d'articles de pédagogie de la religion. On les retrouve, intitulés: «Le Catéchisme pour la vie», dans les numéros de septembre à avril 1944. En octobre de la même année, elle rédige pour la revue La Vie des Communautés religieuses, «La mystique de l'enseignement religieux». En 1946, le R. Père Delcuve, fondateur de Lumen Vitae, sollicite sa collaboration pour l'un des premiers numéros de sa revue. Elle ne refuse rien quand il s'agit de catéchisme. Elle l'a promis. Elle a prononcé de nombreuses conférences, tantôt pour les professeurs d'École Normale, tantôt pour les directeurs d'écoles élémentaires. Elle a recueilli l'admiration de plusieurs et bon nombre d'enseignants ont suivi sa méthode.

\section{Quelle influence a-t-elle exercée?}

Le 24 août 1949, décède Soeur Saint-Ladislas. Elle avait connu des ovations, des succès, elle avait fait salle comble à l'occasion de ses confé-

\footnotetext{
${ }^{15}$ Albini Lafortune à Soeur Saint-Ladislas, 31 mars 1945. AMMN.
} 
rences et de ses cours à l'Université Laval, à Québec. Elle ne verra pas sur terre l'ampleur de l'influence exercée par son oeuvre de catéchète moderne. Les Directives pédagogiques générales du programme de religion paraissent en 1951. Dans ces Directives, elle a introduit les principes de l'école active: "c'est la vie qu'il nous faut»; elle a appelé les élèves à collaborer à leur éducation chrétienne; elle a fourni aux éducateurs les moyens de retenir l'attention des jeunes: récits, dessins, chants, gravures, jeux, fêtes du catéchisme. Elle a proposé de toujours maintenir le contact entre la vie profane et la vérité religieuse enseignée. Elle a introduit des thèmes nouveaux dans la catéchèse: elle parle de formation sociale, de patriotisme, du mystère de la vie selon les desseins de Dieu. Son éditeur lui envoie un mot d'admiration au sujet de sa leçon sur le patriotisme. M. Clément Marchand apprécie de la façon suivante:

Votre leçon sur le patriotisme compte parmi les plus belles choses que l'on ait écrites chez nous sur ce sujet. La première partie surtout est d'une force qui entraine l'adhésion complète de l'esprit et il s'en dégage une émotion féconde.

Je crois que cette leçon devrait être lue dans le texte au milieu d'un grand silence. Comment de jeunes coeurs pourraient-ils ne pas vibrer en écoutant ce commentaire vivant où l'on voit mieux de quel prix la notion de patrie a été payée par nos ancêtres. Des pages comme celles-là dépassent les cadres de l'ouvrage et devront un jour figurer dans une anthologie de prosateur. Je les préfère et de beaucoup à certains morceaux académiques que l'on trouve dans l'oeuvre de nos historiens ${ }^{16}$.

Soeur Saint-Ladislas et après elle, les auteurs du catéchisme de 1951, ont voulu un enseignement religieux qui dépasse «la sphère de la spiritualité individuelle, car la pensée catholique prend de l'homme une vue assez haute et assez large pour ordonner en même temps tous les aspects de sa vie collective, sociale et politique» ${ }^{17}$.

De l'avis des historiens, les années 1935-1950 marquent un progrès dans l'enseignement religieux; Aux Petits du Royaume y joue un rôle novateur. Le remplacement du Catéchisme des provinces en 1951 est un progrès en partie dû aux Directives de 1948. De même une Méthodologie spéciale parue en 1950. Les Directives, émanées du Département de l'Instruction Publique, en 1959, reprennent les idées de Soeur SaintLadislas; on y a ajouté ce sur quoi elle avait le plus insisté au Comité de religion: que les catéchètes fassent entendre la Parole de Dieu à travers l'Écriture Sainte, la Doctrine de l'Église et la Liturgie. La Parole entendue

16 Clément Marchand à Soeur Saint-Ladislas, 19 janvier 1943. AMMN.

${ }^{17}$ Anita Sylvestre, Une pionnière du renouveau catéchistique. Montréal, Institut pédagogique, 1963, p. 75 (texte dactylographié). 
est un appel, la réponse du catéchisé s'exprime par la prière. la Liturgie et la vie morale. La méthode demeure la même, inductive, intégrale: la leçon se développe suivant les mêmes étapes.

En 1952, la Commission épiscopale de l'enseignement religieux créait un organisme officiel de coordination des projets et des travaux en matières religieuses. Dans un rapport de cet organisme, on fait remonter l'origine du renouveau vers 1935 ; on nomme comme précurseurs $\mathbf{M}^{\mathrm{gr}}$ Irénée Lussier, le Père Aĺcantara Dion, Soeur Saint-Ladisias ei $\mathbf{M}^{\mathrm{q}}{ }^{\mathrm{er}} \mathbf{j}$.-Mi. Coderre, président de l'Office catéchistique provincial.

L'influence de Soeur Saint-Ladislas et de son oeuvre a traversé les continents. Des commandes de catéchisme de l'année 1961-1962 sont venues de l'immense continent africain et des pays suivants: Philippines, Inde, Grèce, Norvège, Ceylan, Italie, Portugal, Belgique, et Colombie. Le Japon a traduit son oeuvre, elle circule dans les écoles catholiques.

Les limites de ce travail ne nous permettent pas de citer tous les témoignages de l'influence de son oeuvre à travers le monde. Mais nous qui l'avons vue et entendue, nous savons que Soeur Saint-Ladislas voulait être la petite étincelle, allumeuse de l'amour effectif de Jésus-Christ dans le monde entier. À la dernière page de son livre Catéchisme et vie chrétienne, Soeur Saint-Ladislas adresse une invitation à tous les catéchètes canadiens: celle d'apporter leur collaboration à la solution du problème catéchistique tel que posé dans la province de Québec et dans son rapport avec la vie chrétienne. Se rappelant sans doute l'ultime désir de notre grande catéchète, des Soeurs de notre Institut ont continué son travail, dans le même esprit de service et pour continuer le charisme initial de notre Congrégation.

Soeur Saint-Ladislas a fort préconisé une méthode, mais elle a reconnu aussi la limite de toute méthode et de tout manuel s'ils n'étaient animés par des catéchètes qui portent au coeur la flamme sacrée.

\section{Conclusion}

Après la fondation de l'Office provincial de catéchèse en 1952, les étapes du renouveau se précipitent: sessions d'études pour les prêtres et les éducateurs, publications nombreuses, séries de conférences et de cours souvent animés par des spécialistes européens, multiplication des offices diocésains de catéchèse, fondations de l'Institut supérieur de Sciences reli- 
gieuses de l'Université de Montréal, de l'Institut catéchétique de l'Université Laval et de l'Institut dominicain de pastorale. Des membres de diverses Communautés religieuses ont fréquenté ces Institutions afin de donner à l'Église un plus grand nombre d'éducateurs de la foi. La Congrégation des Soeurs de l'Assomption de la Sainte Vierge, pour continuer l'oeuvre commencée au début de son histoire, envoya ses éducatrices dans les écoles du renouveau de l'enseignement religieux, même en Europe. Nos catéchètes, avec les moyens perfectionnés de notre époque, construisent elles aussi des instruments de travail en vue d'éduquer la foi: Soeur Alice Mignault, Soeur Gabrielle Roussel, Soeur Colette Vaugeois, Soeur Irène Paquette.

Nous formons des voeux pour que l'Église trouve des éducateurs qui, comme hier Soeur Saint-Ladislas, étudient aujourd'hui les documents de notre époque, tel La catéchèse en notre temps, de Jean-Paul II et qu'ils produisent des instruments de travail, à la portée de tous, étincelles capables de rallumer le feu sous la cendre; et que, modelé par l'Église au début de notre histoire, le beau visage de la foi catholique revive, au pays de Québec, dans les arts; dans les Lettres, dans la vie de tous, parce qu'il aura des apôtres, des prophètes et des saints. 